\title{
An efficient technique for Bayesian modeling of family data using the BUGS software
}

\author{
Harold T. Bae ${ }^{1 *}$, Thomas T. Perls ${ }^{2}$ and Paola Sebastiani ${ }^{3}$ \\ ${ }^{1}$ School of Biological and Population Health Sciences, College of Public Health and Human Sciences, Oregon State University, Corvallis, OR, USA \\ ${ }^{2}$ New England Centenarian Study, Department of Medicine, Boston University School of Medicine, Boston, MA, USA \\ ${ }^{3}$ Department of Biostatistics, Boston University School of Public Health, Boston, MA, USA
}

\section{Edited by:}

Mariza De Andrade, Mayo Clinic,

USA

Reviewed by:

Qingzhao Yu, Louisiana State

University Health Sciences Center,

USA

Guimin Gao, Virginia

Commonwealth University, USA

*Correspondence:

Harold T. Bae, School of Biological and Population Health Sciences,

College of Public Health and Human

Sciences, Oregon State University,

Milam 151, Corvallis, OR 97331 ,

USA

e-mail: harold.bae@oregonstate.edu
Linear mixed models have become a popular tool to analyze continuous data from family-based designs by using random effects that model the correlation of subjects from the same family. However, mixed models for family data are challenging to implement with the BUGS (Bayesian inference Using Gibbs Sampling) software because of the high-dimensional covariance matrix of the random effects. This paper describes an efficient parameterization that utilizes the singular value decomposition of the covariance matrix of random effects, includes the BUGS code for such implementation, and extends the parameterization to generalized linear mixed models. The implementation is evaluated using simulated data and an example from a large family-based study is presented with a comparison to other existing methods.

\section{Keywords: BUGS, parameterization, family-based study, covariance matrix, linear mixed models}

\section{INTRODUCTION}

Many observational studies are designed using some form of clustered sampling that introduces correlation between observations within the same cluster. A popular study design that produces correlated observations is the family-based study (Ott et al., 2011), in which families (or clusters) may be selected because family members are enriched of some particular trait of interest. In this design, multiple relatives within the same family are enrolled in the study, and subjects from the same family cannot be assumed independent because they share some genetic background and may have more similar phenotypes than members from different families. In this context, standard statistical methods that assume independent and identically distributed observations are not appropriate because ignoring the correlation between observations may impact the false positive rates of statistical methods (Cannon et al., 2001).

When the trait of interest is continuous, a linear mixed-effects model can be used to account for the family structure by using random effects with a variance-covariance matrix that describes the within and between family covariances. However, the implementation of such models in the BUGS software (Lunn et al., 2000) becomes challenging due to the high dimensionality of the random effects vector, which is as large as the sample size in the study. The fact that the high-dimensional covariance matrix can only be updated as a composite whole in BUGS (Burton et al., 1999) increases the computational burden of the Markov Chain Monte Carlo (MCMC) estimation and often results in a failure to compile the model. To tackle this implementation issue, Waldmann (2009) have proposed an approach based on a decomposition of the multivariate normal distribution of the random effects into univariate normal distributions using conditional distributions (Hallander et al., 2010). Our experience with this approach is that it fails to produce accurate results with large multigenerational families.

This paper describes an alternative parameterization that uses the singular value decomposition of the large covariance matrix of the random effects to fit a mixed model with independent random effects and avoids the use of large variance matrices. This approach is not novel and, for example, it was used in factored spectrally transformed linear mixed models (FaST-LMM) (Lippert et al., 2011) for fast computations with family data. The novelty of our contribution is to use this approach for an efficient implementation in the BUGS software and to extend it to generalized linear models. The BUGS code is also provided and a specific example as well as results from simulation studies are presented with some discussion.

\section{PROPOSED PARAMETERIZATION}

A common parameterization of linear mixed models for correlated family data is:

$$
y \mid \beta, \sigma_{g}^{2}, \sigma_{e}^{2}=X \beta+\rho+\epsilon,
$$

where $y$ is the phenotype vector of size $n \times 1, X$ is the $n \times(p+1)$ design matrix that contains values of measured covariates, $\beta$ is the fixed effect vector of size $p \times 1, \rho$ is the random effect vector of size $n \times 1$, which accounts for the additive polygenic effect, and $\epsilon$ is a vector of random errors of size $n \times 1$. The vectors $\rho$ and $\epsilon$ are marginally independent and follow distributions:

$$
\begin{aligned}
& \rho \sim N\left(\underline{0}, \sigma_{g}^{2} A\right) \\
& \epsilon \sim N\left(\underline{0}, \sigma_{e}^{2} I_{n}\right),
\end{aligned}
$$


where $A$ represents the known additive genetic relationship matrix (see Figure 1), $\sigma_{g}^{2}$ is the genetic variance, and $\sigma_{e}^{2}$ is the error variance (Eu-ahsunthornwattana et al., 2014). Under this parameterization the variance-covariance matrix of the observation $y$ is the matrix:

$$
\operatorname{Var}\left(y \mid \beta, \sigma_{g}^{2}, \sigma_{e}^{2}\right)=\sigma_{g}^{2} A+\sigma_{e}^{2} I_{n} .
$$

One can estimate the narrow-sense heritability of a trait as a ratio of the genetic variance $\sigma_{g}^{2}$ to the total phenotypic variance $\sigma_{g}^{2}+$ $\sigma_{e}^{2}$, such that $h^{2}=\frac{\sigma_{g}^{2}}{\sigma_{g}^{2}+\sigma_{e}^{2}}$.

In a recent review article, Muller et al. (2013) described the following parameterization for general linear mixed-effects models:

$$
y \mid \beta, \sigma_{g}^{2}, \sigma_{e}^{2}=X \beta+G u+\epsilon
$$

where $u \sim N\left(\underline{0}, \sigma_{g}^{2} I_{s}\right), e \sim N\left(\underline{0}, \sigma_{e}^{2} I_{n}\right)$, and $G$ is a matrix of known coefficients. The advantage of the parameterization in Equation (3) is that the random effects $u_{i}, i=1, \ldots, n$ are marginally independent rather than being correlated as in the initial parameterization.

The two parameterizations are equivalent once the matrix $G$ is derived from the singular value decompostion of the matrix

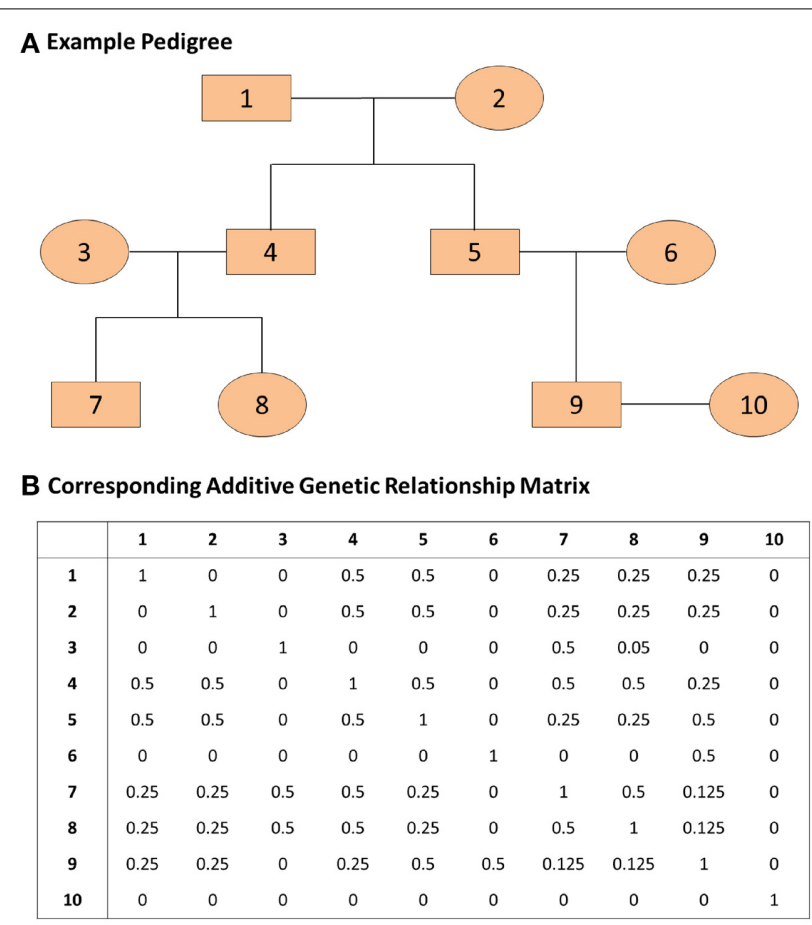

FIGURE 1 | An example pedigree and corresponding additive genetic relationship matrix. (A) The pedigree on the top panel displays the relations among family members. (B) The additive genetic relationship matrix is the kinship matrix multiplied by 2 ; the kinship matrix contains kinship coefficients between any pair of family members and these coefficients represent the probability that two individuals share the same gene allele by identity by descent. The covariance between two family members $i$ and $j$ with kinship coefficient $k_{i j}$ is $2 k_{i j} \sigma_{g}^{2}$ where $\sigma_{g}^{2}$ represents the genetic variance.
A. Specifically, by setting $A=U S^{1 / 2} S^{1 / 2} U^{T}$ and letting $G=$ $U S^{1 / 2}$, the variance-covariance matrix of $y$ from the model in Equation (3) is

$$
\begin{aligned}
\operatorname{Var}(y \mid \beta, u, e) & =\sigma_{g}^{2} G G^{T}+\sigma_{e}^{2} I_{n}=\sigma_{g}^{2} U S^{1 / 2} S^{1 / 2} U^{T}+\sigma_{e}^{2} I_{n} \\
& =\sigma_{g}^{2} A+\sigma_{e}^{2} I_{n}
\end{aligned}
$$

Note that the matrix $U S^{1 / 2}$ needs to be computed only once. We provide an example R script that computes $U S^{1 / 2}$ given a familybased data set in the Supplementary Material.

The parameterization can be extended to generalized linear mixed models. In a generalized linear mixed model (GLMM), the formulation becomes:

$$
\begin{array}{r}
y \mid \beta, \phi, \sigma_{g}^{2} \sim \mathcal{D} \in \text { exponential family } \\
E\left(y \mid \beta, \phi, \sigma_{g}^{2}\right)=g(\eta) \\
\eta=X \beta+\rho,
\end{array}
$$

where $\phi$ is the dispersion parameter of the distribution belonging to the exponential family and $g(\cdot)$ is the link function. The parameterization of the random effects applies as before and the linear predictors include the random effects in addition to the fixed effects. The difference here is the assumption that the observations are independent, conditionally on the random effects.

\section{A REAL DATA EXAMPLE}

As an example to illustrate the implementation in OpenBUGS, we consider the task of estimating the heritability of transferrin receptor levels from a large family-based study. The data are from the Long Life Family Study (LLFS) that between 2006 and 2009 enrolled approximately 5000 individuals from 583 families demonstrating clustering for longevity and healthy aging in the United States and Denmark (Sebastiani et al., 2009; Newman et al., 2011). A typical family of the LLFS includes a proband, the proband's siblings, their spouses, offspring of probands and siblings, and their spouses. The family size varies between 3 individuals to 77 individuals. In this example, transferrin receptor levels were adjusted for age at enrollment in the study and insulin levels. The kinship matrix $A$ was calculated with the $\mathrm{R}$ package kinship2 (Therneau et al., 2012) and the $\mathrm{R}$ code to generate the matrix $G$ is provided in Supplementary Material.

The entire BUGS code is shown with some comments below. There are a few points that are noteworthy. First, the matrix $G$, which is computed within $\mathrm{R}$, is part of the BUGS data input. The calculation of the matrix $G$ is required only once. Second, the variable offset is used to indicate the individuals who belong to each specific family. For example, in the code below, we use the index $i$ to represents families and the index $j$ to represent individuals. The first few values of the variable offset in this particular example are $c(1,8,16, \ldots)$. When $i=1$ (the first family), $j$ will span from 1 to 7 , indicating that individuals 1 through 7 belong to family 1 . When $i=2$ (the second family), $j$ will span from 8 to 15 , indicating that individuals 8 through 15 belong to family 2 . Then, 
based on the values of $j$ and offset, the inner product between an appropriate row of the matrix $G$ and vector $u$ is computed.

A total of 11,000 iterations with the first 1000 as a burn-in was sufficient to reach the convergence of the estimates. On average, each iteration took $0.0892 \mathrm{~s}$ on an Intel(R) Core i5 processor $(2.53 \mathrm{GHz})$ with $4 \mathrm{~GB}$ of RAM. The data were also analyzed using the method proposed in Hallander et al. (2010) and by fitting classical linear mixed models with the function lmekin() in coxme (Therneau, 2009) package in R.

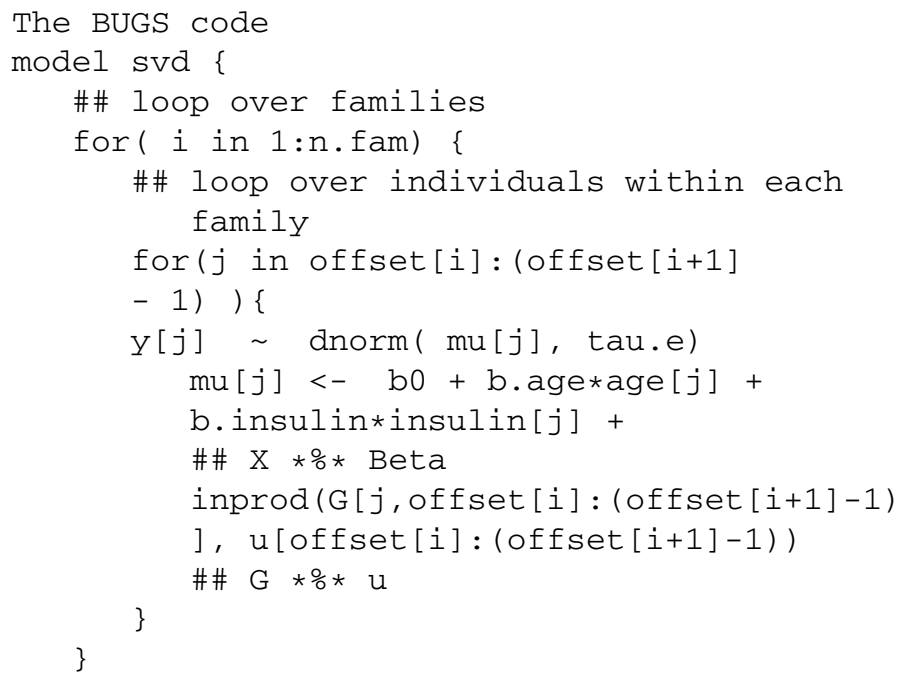

Table 1 compares the point estimates and standard errors (SE) of regression parameters, the variance parameters, and the heritability estimates from the linear mixed models computed using the lmekin() function in R, the proposed method (SVD Model), and the method in Hallander et al. (2010) (conditional Model). Figure 2 displays the posterior distribution of heritability, residual variance, and genetic variance. The point estimates and SE from the R ouput and SVD model are nearly identical. The heritability estimates in the two methods are 0.3677 and 0.3677 , respectively, with a difference of only 0.0031 . However, compared to these two methods, the conditional model produces discrepant results; the residual variance is over-estimated and the genetic variance is under-estimated, which leads to inconsistent estimate of the heritability and the 95\% credible intervals of the two Bayesian methods do not overlap. Inconsistent results between the SVD model and conditional model are surprising since, in theory, both approaches rely on decomposition methods that should lead to exactly the same covariance matrix. To further investigate this discrepancy, simulations of several scenarios of extended pedigree data structures were performed (see the next section for details on simulations). However, we were not able to pinpoint the reason for the apparent discrepancy. The advantage of the Bayesian approach here, compared to the classical approach is to provide measures of the uncertainity of the heritability estimate by the posterior distribution (Figure 2) and the 95\% credible interval.

We extended our approach to logistic regression with family data. To our knowledge, no statistical package fully adjusts for the familiar relatedness when the outcome variable is binary. A commonly used approach is to fit logistic regression with one random effect per family or use a generalized estimating equation (GEE), in which each family is considered a cluster. Thus, there is a need to develop such methods to analyze binary traits coming from family data. As an example, we again used the data from the LLFS, in which the binary trait was the occurrence of cardiovascular diseases within 8 years of follow-up and covariates were sex and age at enrollment of participants. Cardiovascular diseases were defined as having any one of the following: myocardial infarction, coronary artery bypass grafting, congestive heart failure, and atrial fibrillation (Sebastiani et al., 2013). We modified the BUGS code by changing the response variable $y[j]$ to follow a Bernoulli distribution and modeling the random effects vector on the logodds scale. For comparison, a logistic regression model based on the GEE approach was also performed. Table 2 shows the point estimates, standard errors, and 95\% credible intervals based on the two approaches. The point estimates between the two methods are comparable, although the standard errors from the GEE model are slightly smaller for all three fixed effects parameters. This is expected, as our proposed model takes into account the full kinship matrix, whereas the GEE model treats each family as a single cluster. It is also noteworthy to point out that convergence can be slow for implementing this parameterization in a logistic regression framework. A good heuristic for faster convergence is to start with the maximum likelihood estimates of the fixed effects parameters and then try to estimate the genetic variance $\sigma_{g}^{2}$.

\section{EMPIRICAL EVALUATION}

A simulation study was conducted to evaluate the accuracy of the implementation of our method in different types of family structure for normal data. Four different scenarios, in which the current implementation was evaluated, are as follows:

- Nuclear Family: This is the simplest family structure in which there is a couple with two offspring. There were a total of 100 such families, which led to $N=400$.

- Two-trios: This is the simplest form of extended pedigree structure with first-, second-, and third-degree relatives where two parent-offspring trios are related through a sibling pair in the 
Table 1 | Comparison of Point Estimates (PE), standard errors (SE), and 95\% Credible Intervals (95\% Cl) for continuous trait.

\begin{tabular}{|c|c|c|c|c|c|c|c|c|}
\hline & \multicolumn{2}{|c|}{ R (Imekin) } & \multicolumn{3}{|c|}{ SVD model } & \multicolumn{3}{|c|}{ Conditional model } \\
\hline Age & 0.0101 & 0.0008 & 0.0102 & 0.0009 & $0.0084-0.0119$ & 0.0101 & 0.0009 & $0.0082-0.0119$ \\
\hline Insulin & 0.0021 & 0.0004 & 0.0022 & 0.0004 & $0.0014-0.0030$ & 0.0022 & 0.0004 & $0.0014-0.0030$ \\
\hline Heritability & 0.3677 & $\mathrm{~N} / \mathrm{A}$ & 0.3707 & 0.0345 & $0.3015-0.4402$ & 0.1325 & 0.0257 & $0.0957-0.195$ \\
\hline
\end{tabular}

$R$ (Imekin), results obtained from using Imekin function in R; SVD Model, results obtained from using the proposed method based on singular value decomposition of the additive genetic relationship matrix; Conditional Model, results obtained from using the method in Hallander et al. (2010). The total sample size was 4229 with 558 unique families.
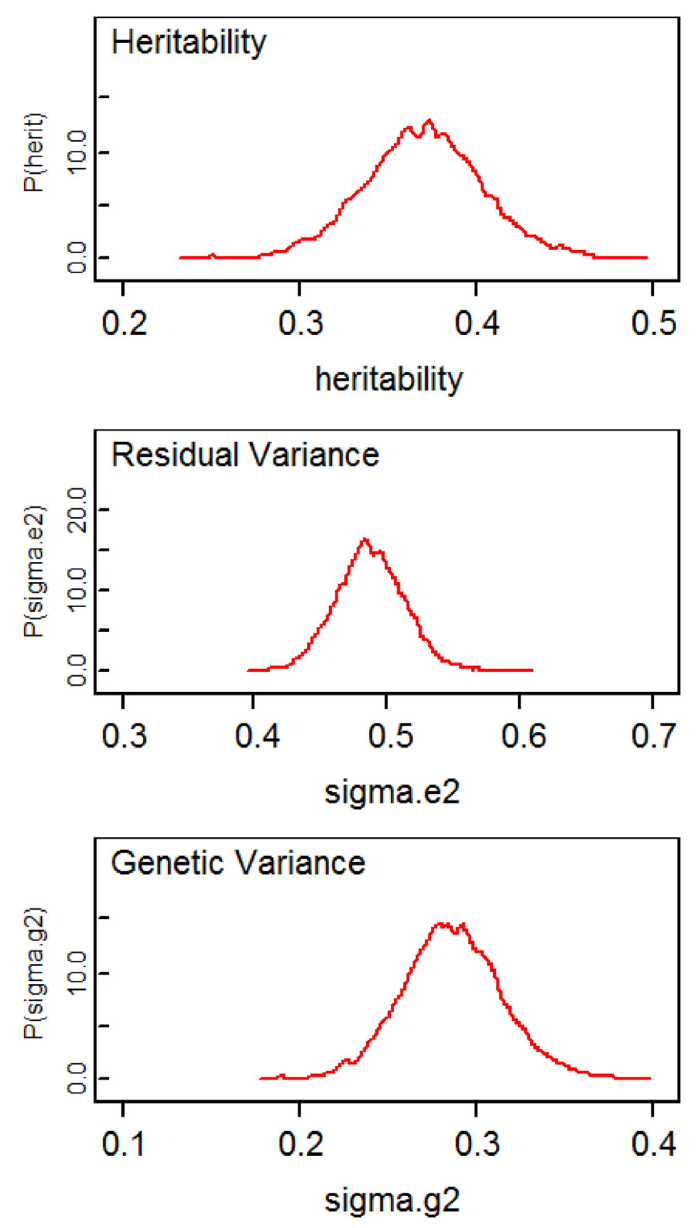

FIGURE 2 | Plots of posterior distributions of heritability, residual variance, and genetic variance.

parent generation. There were a total of 100 such families and the total sample size was 600 .

- Asymmetric Family: This is an asymmetric version of the second scenario, in which the first trio has only one offspring and the second trio has ten offspring. There were a total of 100 such families with a total sample size of 1500 .
- Combination: This is a combination of the first and second scenario with several offspring in the second scenario. The total sample size was 1400 .

To generate correlated data, a kinship matrix from each family $K_{f}$ was created, and the variance-covariance matrix of the observations was defined as $V=\sigma_{e}^{2} I_{n}+2 \sigma_{g}^{2} \operatorname{diag}\left(K_{1}, K_{2}, \ldots, K_{n f}\right)$, where $n$ is the total sample size and $n_{f}$ is the number of families in each scenario. In each simulation, a vector $Z$ of independent and normally distributed observations was generated and transformed into $Y=U D^{1 / 2} Z$ where $U$ and $D$ are the matrix of eigenvectors and eigenvalues from the spectral decomposition of the variance-covariance matrix $V$. This transformation guarantees that $V(Y)=U D^{1 / 2} V(Z) D^{1 / 2} U^{T}=V$ so that the simulated data have the desired correlation.

Table 3 compares the point estimates and standard errors of the variance components from the linear mixed models computed using the lmekin() function in R, the proposed method (SVD Model), and the method in Hallander et al. (2010) (conditional Model). In all scenarios, there was no discernible difference between the estimates among the three approaches, which suggests that the implementation in BUGS works correctly.

\section{CONCLUSION}

The proposed BUGS code provides an easy and efficient way to account for extended family structures in linear mixed models. Results from a real data set as well as simulation data show that this implementation produces consistent results with the classical linear mixed models in $\mathrm{R}$. The usefulness of this approach is that it allows for linear mixed modeling of family-based data in the BUGS software, and thus possibly facilitates the use of Bayesian modeling of family-based data. The advantage of the Bayesian approach is that it provides an estimate of heritability but implementation is often challenging. We also illustrate the extension of our approach to generalized linear models that can be efficiently implemented in BUGS.

\section{ACKNOWLEDGMENTS}

This work was funded by the National Institute on Aging (NIA U19-AG023122, U01-AG023755 to Thomas T. Perls), the National Heart Lung Blood Institute (R21HL114237 to Paola 
Table 2 | Comparison of Point Estimates (PE), Standard Errors (SE), and 95\% Credible Intervals (95\% Cl) for binary trait.

\begin{tabular}{|c|c|c|c|c|c|c|}
\hline & \multicolumn{3}{|c|}{ SVD model } & \multicolumn{3}{|c|}{ GEE model } \\
\hline Sex & -0.470 & 0.077 & $-0.621--0.318$ & -0.458 & 0.075 & $-0.604--0.311$ \\
\hline Age & 0.064 & 0.0026 & $0.058-0.069$ & 0.062 & 0.0024 & $0.057-0.066$ \\
\hline
\end{tabular}

SVD Model, results obtained from using the proposed method based on singular value decomposition of the additive genetic relationship matrix in a logistic regression; GEE Model, results from generalized estimating equations in a logistic regression. The total sample size was 4654 with 583 unique families.

Table 3 | Comparison of Point Estimates (PE) and Standard Errors (SE) of variance components in simulated data.

\begin{tabular}{|c|c|c|c|c|c|c|c|}
\hline & & \multicolumn{2}{|c|}{ R (Imekin) } & \multicolumn{2}{|c|}{ SVD model } & \multicolumn{2}{|c|}{ Conditional model } \\
\hline Nuclear family & $\sigma_{g}^{2}$ & 0.734 & $\mathrm{~N} / \mathrm{A}$ & 0.738 & 0.211 & 0.741 & 0.206 \\
\hline Two-trios & $\sigma_{e}^{2}$ & 0.936 & N/A & 0.926 & 0.193 & 0.936 & 0.200 \\
\hline \multirow[t]{2}{*}{ Asymmetric family } & $\sigma_{e}^{2}$ & 0.963 & N/A & 0.961 & 0.090 & 0.956 & 0.087 \\
\hline & $\sigma_{g}^{2}$ & 1.024 & N/A & 1.029 & 0.143 & 1.043 & 0.141 \\
\hline \multirow[t]{2}{*}{ Combination } & $\sigma_{e}^{2}$ & 1.030 & N/A & 1.028 & 0.117 & 1.031 & 0.123 \\
\hline & $\sigma_{g}^{2}$ & 1.915 & N/A & 1.927 & 0.180 & 1.923 & 0.186 \\
\hline
\end{tabular}

$R$ (Imekin), results obtained from using Imekin function in R; SVD Model, results obtained from using the proposed method based on singular value decomposition of the additive genetic relationship matrix; Conditional Model: results obtained from using the method in Hallander et al. (2010).

Sebastiani), and the National Institure of General Medical Sciences T32GM074905.

\section{SUPPLEMENTAL DATA}

Sample R script for computing the singular value decomposition of the additive genetic relationship matrix.

\#\# Read the Phenotype Data \#\#

pheno.file <- read.csv("pheno.data.csv")

\#\# Read the Pedigree Data

ped.file <- read.csv("ped.data.csv")

\#\# Variable Description:

\#\# subject = unique individual ID

\#\# gpedid = family $I D$

\#\# dadsubj = ID of the father

\#\# momsubj = ID of the mother

\#\# sex $=$ sex of individual

\#\# Needs to be ordered by family

ped.file <- ped.file[ order(ped.file\$pedid), ]

\#\# Use package kinship2 to construct the pedigree object and

\#\# the kinship matrix.

library (kinship2)

\#\# This creates pedigree objects

mped.full <- pedigree(id=ped.file\$subject,

dadid=ped.file\$dadsubj,

momid=ped.file\$momsubj, sex=ped.file\$sex, famid=ped.filesgpedid, missid=0)

\#\# This creates the kinship matrix

kmat.full <- kinship (mped.full)

\#\# Singular Value Decomposition by family

family.list <- intersect (unique (ped.file\$gpedid), unique (pheno.file\$gpedid))

my. U <- vector ("list")

my.S <- vector("list")

my.kmat <- vector("list")

my.G <- vector("list")

for (i in 1:length (family.list)) \{

$\mathrm{p}<-$ ped.file[ which(ped.file\$gpedid

$==$ family.list $[i])$,

mped <- pedigree $(i d=p \$ s u b j e c t, ~ d a d i d=p \$ d a d s u b j$, momid=p\$momsubj, sex=p\$sex, famid $=$ p\$gpedid, missid=0)

kmat <- $2 *$ kinship (mped)

\#\# Now get kinship submatrix for subjects

ind.subj <- match(pheno.file\$subject

[which (pheno.file\$gpedid ==

family.list[i])], row.names(kmat))

test <- svd ( kmat[ind.subj[ which(is.na (ind. subj) $==F)$ ],

ind.subj [ which(i.s.na (ind.subj)==F)] ])

my.U[[i] $]<-$ test\$u

my.S[[i] $]<-$ test\$d

my.G[[i]] <- test\$u \%*\% diag ( $\operatorname{sqrt}($ test\$d))

my. kmat [i]] $<-$ kmat [ind.subj

[ which (is.na (ind.subj) ==F)], ind.subj [ which(is.na (ind.subj) $==F)]$ ] 


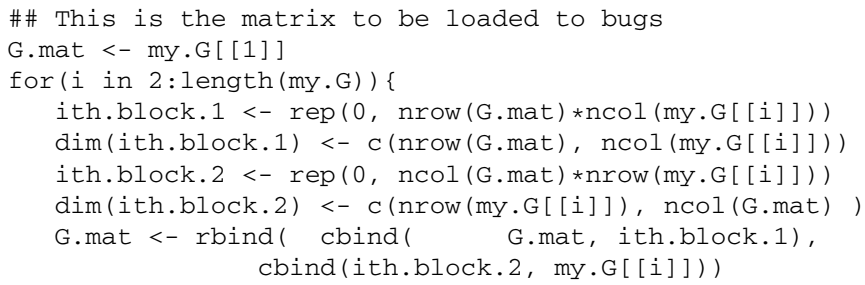

\section{REFERENCES}

Burton, P. R., Tiller, K. J., Gurrin, L. C., Cookson, W. O., Musk, A. W., and Palmer, L. J. (1999). Genetic variance components analysis for binary phenotypes using generalized linear mixed models (glmms) and gibbs sampling. Genet. Epidemiol. 17, 118-140. doi: 10.1002/(SICI)1098-2272(1999)17:2<118::AIDGEPI3 > 3.0.CO;2-V

Cannon, M. J., Warner, L., Taddei, J. A., and Kleinbaum, D. G. (2001). What can go wrong when you assume that correlated data are independent: an illustration from the evaluation of a childhood health intervention in brazil. Stat. Med. 20, 1461-1467. doi: 10.1002/sim.682

Eu-ahsunthornwattana, J., Miller, E. N., Fakiola, M., Consortium, W. T. C. C., Jeronimo, S. M. B., Blackwell, J. M., et al. (2014). Comparison of methods to account for relatedness in genome-wide association studies with family-based data. PLOS Genet. 10:e1004445. doi: 10.1371/journal.pgen. 1004445

Hallander, J., Waldmann, P., Wang, C., and Sillanpaa, M. J. (2010). Bayesian inference of genetic parameters based on conditional decompositions of multivariate normal distributions. Genetics 185, 645-654. doi: 10.1534/genetics.110. 114249

Lippert, C., Listgarten, J., Liu, Y., Kadie, C. M., Davidson, R. I., and Heckerman D. (2011). Fast linear mixed models for genome-wide association studies. Nat. Methods 8, 833-835. doi: 10.1038/nmeth.1681

Lunn, D. J., Thomas, A., Best, N., and Spiegelhalter, D. (2000). Winbugs - a bayesian modelling framework: Concepts, structure, and extensibility. Stat. Comput. 10, 325-337. doi: 10.1023/A:1008929526011

Muller, S., Scealy, J. L., and Welsh, A. H. (2013). Model selection in linear mixed models. Stat. Sci. 28, 135-167. doi: 10.1214/12-STS410
Newman, A. B., Glynn, N. W., Taylor, C. A., Sebastiani, P., Perls, T. T., Mayeux, R., et al. (2011). Health and function of participants in the long life family study: a comparison with other cohorts. Aging (Albany NY) 3, 63-76.

Ott, J., Kamatani, Y., and Lathrop, M. (2011). Family-based designs for genomewide association studies. Nat. Rev. Genet. 12, 465-474. doi: 10.1038/nrg2989

Sebastiani, P., Hadley, E. C., Province, M., Christensen, K., Rossi, W., Perls, T. T., et al. (2009). A family longevity selection score: ranking sibships by their longevity, size, and availability for study. Am. J. Epidemiol. 170, 1555-1562. doi: 10.1093/aje/kwp309

Sebastiani, P., Sun, F. X., Andersen, S. L., Lee, J. H., Wojczynski, M. K., Sanders, J. L., et al. (2013). Families enriched for exceptional longevity also have increased health-span: findings from the long life family study. Front. Public Health 1:38. doi: $10.3389 /$ fpubh.2013.00038

Therneau, T. (2009). coxme: Mixed Effects Cox Models. Available online at: http:// cran.r-project.org/web/packages/coxme/coxme.pdf.

Therneau, T., Atkinson, E., Sinnwell, J., Matsumoto, M., Schaid, D., and Mcdonnell, S. (2012). kinship2: Pedigree Functions. Available online at: http://cran.r-project. org/web/packages/kinship2/kinship2.pdf.

Waldmann, P. (2009). Easy and flexible bayesian inference of quantitative genetic parameters. Evolution 63, 1640-1643. doi: 10.1111/j.1558-5646.2009.00645.x

Conflict of Interest Statement: The authors declare that the research was conducted in the absence of any commercial or financial relationships that could be construed as a potential conflict of interest.

Received: 11 August 2014; accepted: 24 October 2014; published online: 18 November 2014

Citation: Bae HT, Perls TT and Sebastiani P (2014) An efficient technique for Bayesian modeling of family data using the BUGS software. Front. Genet. 5:390. doi: 10.3389/ fgene.2014.00390

This article was submitted to Statistical Genetics and Methodology, a section of the journal Frontiers in Genetics.

Copyright (c) 2014 Bae, Perls and Sebastiani. This is an open-access article distributed under the terms of the Creative Commons Attribution License (CC BY). The use, distribution or reproduction in other forums is permitted, provided the original author(s) or licensor are credited and that the original publication in this journal is cited, in accordance with accepted academic practice. No use, distribution or reproduction is permitted which does not comply with these terms. 